\title{
Experience of operative treatment in 27 patients with intraspinal neurilemmoma
}

\author{
HAIBO LI* ${ }^{*}$, YIPING WENG ${ }^{*}$, DONG ZHOU, LUMING NONG and NANWEI XU \\ Department of Orthopaedics, The Second People's Hospital of Changzhou, \\ Affiliated to Nanjing Medical University, Changzhou, Jiangsu 213003, P.R. China
}

Received June 28, 2017; Accepted August 21, 2017

DOI: $10.3892 / \mathrm{ol} .2017 .6799$

\begin{abstract}
To investigate the clinical outcomes of posterior laminectomy on intraspinal neurilemmoma. Twenty-seven patients with intraspinal neurilemmoma admitted to the Second People's Hospital of Changzhou Affiliated to Nanjing Medical University from January 2010 to October 2015 were selected, and they received posterior laminectomy, and there were 5 patients whose nerve root and tumor were removed together because they could not be separated; 17 patients receiving total laminectomy or semi-laminectomy combined with facetectomy on the affected side were treated with spinal dynamic pedicle screw fixation or replantation of lamina or bone graft fusion and internal fixation. The patients were followed up regularly to observe the clinical outcomes after operation. The spinal stability and bone graft fusion were observed via X-ray film in review. Whether there was tumor recurrence was observed via MRI in review according to the individual condition. The operation time was $2.5-8 \mathrm{~h}$ (4.2 $\mathrm{h}$ on average). The intraoperative blood loss was $420-1,500 \mathrm{ml}$ (760 $\mathrm{ml}$ on average). Tumors in 26 patients were totally resected, and tumor in 1 patient was partially resected due to tumor tissue invasion against the anterior sacral vascular plexus. All patients were pathologically confirmed as neurilemmoma after operation. Ten patients suffered from cerebrospinal fluid leakage in different degrees after operation. During the follow-up for 6-75 months after operation, clinical symptoms and neurological functions of all patients were significantly improved; there was 1 case of tumor residual and no recurrence. During the follow-up, the internal fixation in all patients receiving internal fixation of spine was stable with no vertebral instability. Posterior
\end{abstract}

Correspondence to: Dr Luming Nong or Dr Nanwei Xu, Department of Orthopaedics, The Second People's Hospital of Changzhou, Affiliated to Nanjing Medical University, 29 Xinglong Xiang, Tianning, Changzhou, Jiangsu 213003, P.R. China

E-mail: yanhejienaowe@163.com

E-mail: xunanwei73@163.com

*Contributed equally

Key words: intraspinal neurilemmoma, posterior surgery, laminectomy, clinical outcomes laminectomy can achieve satisfactory clinical outcomes in the treatment of intraspinal neurilemmoma. Internal fixation is needed to reestablish spinal stability during operation for those patients with great damage to spinal stability.

\section{Introduction}

Intraspinal tumors are common neurogenic tumors, accounting for approximately $15 \%$ of central nervous system tumors (1). Intraspinal tumors refer collectively to the primary tumors and metastatic tumors that grow in the spinal canal or spinal cord or adjacent tissue structures to spine (such as the spinal dura mater, nerve roots, blood vessels and intraspinal adipose tissue). Intrathecal tumors include the primary tumors and secondary tumors. The primary intraspinal tumors refer to all tumors originated in intraspinal tissue, and secondary intraspinal tumors refer to tumors originated in adjacent tissues outside spinal canal and spread into spinal canal, and malignant tumors originated in distal sites and spread into spinal canal via metastasis, among which neurilemmoma is a kind of common benign tumor in spinal canal $(2,3)$, and due to the slow tumor growth, hidden onset and no specific symptoms and signs, its early diagnosis is more difficult, and some patients are not treated until they are paralyzed. In particular, its initial manifestation in the early stage is the radiating pain caused by affecting the nerve root, so it is often misdiagnosed as coronary heart disease, cholecystitis and appendicitis, and patients even receive the wrong operation, seriously affecting the patient's operative prognosis (4). Thus, the early diagnosis and early surgery are essential. Twenty-seven patients with intraspinal neurilemmoma were admitted to the Second People's Hospital of Changzhou affiliated to Nanjing Medical University from January 2010 to October 2015, achieving good clinical outcomes.

\section{Patients and methods}

Patients. There were 27 patients in the group, including 15 males and 12 females aged 30-81 years with an average age of 53.7 years; there were 4 cases of cervical segment, 6 cases of thoracic segments and 17 cases of lumbar and sacral segments; there were 25 cases of intradural-extramedullary tumor and 2 cases of epidural tumor. The course of disease was 4-36 months with an average duration of 13.5 months. 
Seventeen cases suffered from limb radiating pain accompanied with hypoesthesia or hyperalgesia in different degrees in physical examination; 10 cases suffered from weakness in walking accompanied with decreased muscle strength, positive pathological signs, tendon hyperreflexia or active in physical examination; 2 cases suffered from numbness in saddle area, fecal and urinary dysfunction. This study was approved by the Ethics Committee of the Second People's Hospital of Changzhou. Signed written informed consents were obtained from all participants before the study.

Radiological examination. All patients underwent X-ray, $\mathrm{CT}$ and MRI examinations before operation. X-ray film showed that there were 8 cases of spinal canal enlargement and vertebral posterior compression, and 5 cases of intervertebral foramen expansion and vertebral pedicle narrowing. CT examination showed that the tumors were mostly oval or circular solid lesions, and some were dumbbell-shaped tumors. MRI examination showed 20 cases of intradural extramedullary oval mass with clear border, 6 cases of dumbbell-shaped tumors and 1 case of larger sacral tumor with irregular shape growing to the anterior pelvic cavity. T1-weighted image (T1WI) showed slightly lower signal, equal signal or equally-low mixed signal, and T2-weighted image (T2WI) mostly showed high signal or mixed signal. Sixteen patients underwent MRI enhanced scan, among which 6 cases showed significant enhancement and 10 cases showed irregular edge enhancement. Before operation, 19 patients were clinically diagnosed as neurilemmoma, and another 8 patients had difficulty in diagnosis and diagnosed pathologically after operation (Table I).

Surgical procedures. Under general anesthesia with tracheal intubation, all patients received posterior midline approach in prone position, except 1 case of large sacral tumor receiving combined anterior and posterior approach. Thoracic tumors were positioned using clip before operation. Longitudinal incision was made on skin and subcutaneous tissue with the affected vertebra as the center, and the paravertebral muscles were peeled off to the articular process. Sixteen cases of tumors were significantly biased to one side, so they received semi-laminectomy, and 6 cases of tumors could be exposed using facetectomy on the affected side; 11 cases of tumor involved a large area, so they received total laminectomy, and zygapophyseal joints on both sides were retained as far as possible. Dura mater was cut along the posterior midline to completely expose tumor tissue, followed by blunt dissection using nerve detacher. One to two nerve roots crossed 5 cases of tumors with unclear boundary, so they were totally resected. Sixteen cases of tumors were totally resected due to complete capsule and clear boundary. Five cases of tumors were partially resected due to difficult in total resection. One case of large sacral tumor received combined anterior and posterior approach. Dural sac was closely and continuously sutured using 6-0 non-invasive stitches, and 6 cases of dural tear were sealed using adjacent fascia. Seventeen patients were treated with spinal dynamic pedicle screw fixation or replantation of lamina or bone graft fusion and internal fixation, including 4 cases of cervical vertebra receiving lateral mass fixation, 1 case of thoracic vertebra receiving replantation of lamina,
Table I. Demographic data for the 27 enrolled patients.

Items

Number of cases

Sex

Male/Female

$15 / 12$

Segment

Cervical segment

4

Thoracic segment

6

Lumbosacral segment

Location

Subdural

25

Epidural

2

6 cases of thoracolumbar vertebrae receiving dynamic pedicle screw fixation and 6 cases of lumbosacral vertebrae receiving pedicle screw fixation + posterior-lateral bone graft fusion.

Post-operative treatment. After operation, patients received conventional antibiotics to prevent infection, dehydration and swelling, and anti-inflammatory treatment using hormone. Cervical gear or waist gear was used for protection for 3 months. Patients were followed up regularly after operation, and spinal stability and bone graft fusion were observed via X-ray in the review; whether there was tumor recurrence was observed via MRI in the review according to individual conditions.

\section{Results}

The operation time was $2.5-8 \mathrm{~h}$ ( $4.2 \mathrm{~h}$ on average). The intraoperative blood loss was $420-1,500 \mathrm{ml}$ (760 $\mathrm{ml}$ on average). Tumors in 26 patients were totally resected, and tumor in 1 patient was partially resected due to tumor tissue invasion against the anterior sacral vascular plexus. All patients were pathologically confirmed as neurilemmoma after operation. Ten patients suffered from cerebrospinal fluid leakage after operation, so the position was adjusted, followed by pressure dressing and wound dressing; and the drainage time was prolonged appropriately, and non-vacuum drainage was adopted. There were 3 cases of refractory cerebrospinal fluid leakage, and the wound was sutured in debridement and healed. During the follow-up for 6-75 months after operation, clinical symptoms and neurological functions of all patients were significantly improved. The mean pain VAS score of 17 patients with limb radiating pain was reduced from 8 points on admission to 4 points at discharge. The average muscle strength of 10 patients with limb weakness and walking instability recovered from Level 3 before operation to level 5 in the review at 1 month after operation. Symptoms of 2 patients with numbness in saddle area and fecal and urinary dysfunction disappeared and the function was recovered in the review at 5 months after operation. Partial tumor tissue was left in 1 patient due to the invasion of larger tumor against anterior sacral vascular plexus, and MRI showed that the tumor tissue did not grow in the review at 6 months after operation. There was no recurrence. The internal fixation in patients receiving spinal internal fixation was in place and the fusion in patients 

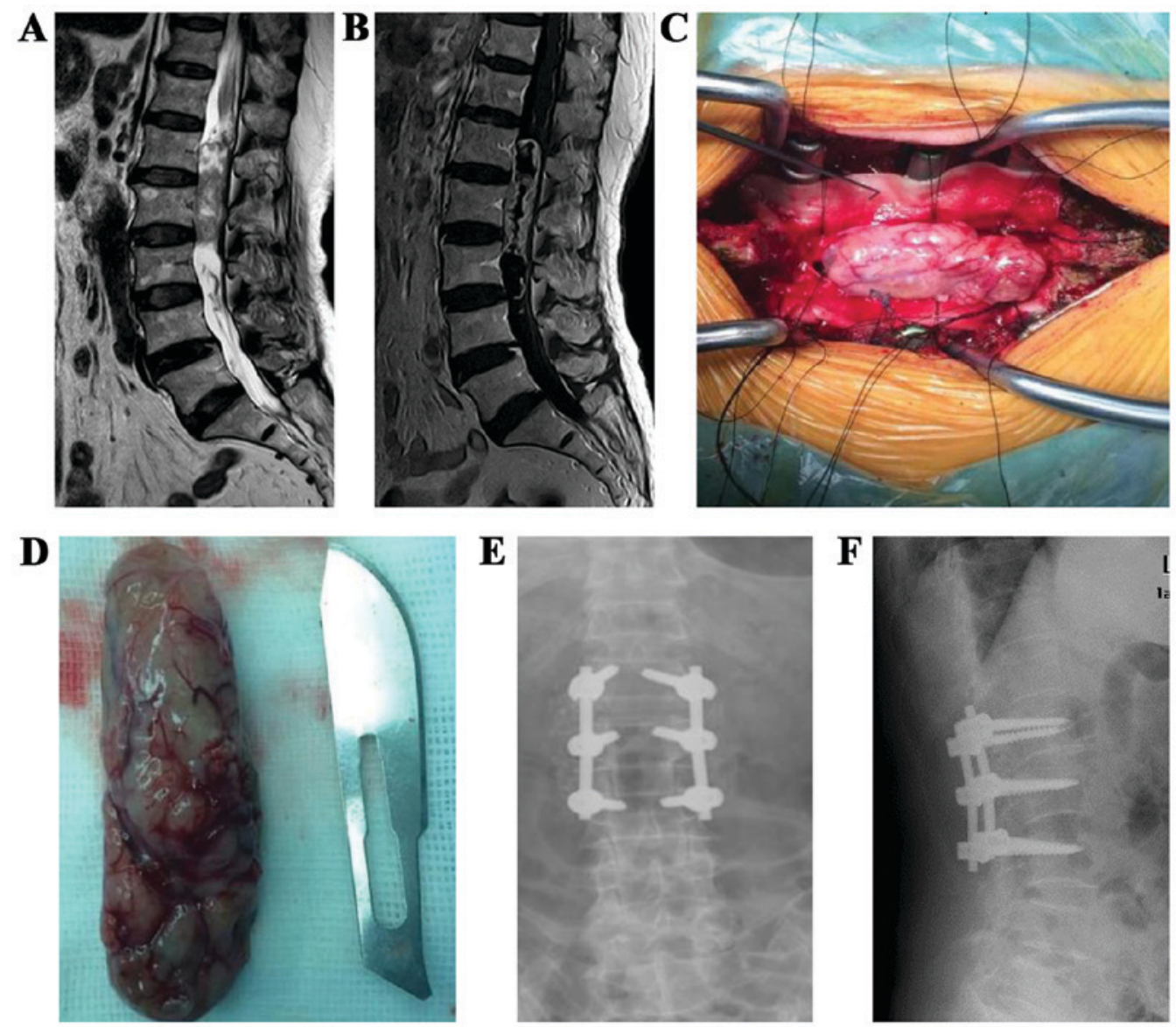

Figure 1. A female patient, 67 years old, neurilemmoma in lumbar spinal canal (L1-L3). (A and B) Pre-operative lumbar MRI showed the intradural extramedullary long oval lesions on L1-L3 segments with smooth edge, T2WI showed mixed high signal (A) and enhanced MRI showed irregular edge enhancement (B). (C and D) Smooth tumor surface and clear boundary during operation, complete excision. (E) Lumbar anteroposterior X-ray film showed the absence of L1-L3 vertebral plate, followed by posterolateral autogenous bone graft. (F) Lumbar lateral X-ray film showed that the internal fixation of pedicle screw was fine in the review at 10 months after operation, and no lumbar instability was found.

receiving fusion surgery was fine. No pedicle prolapse, broken nail or broken rod was found. No spondylolisthesis and instability were found during follow-up (Fig. 1).

\section{Discussion}

Most patients with neurilemmoma are characterized by hidden onset and a longer course of disease. The main symptoms and signs are pain, paresthesia, dyskinesia and sphincter dysfunction. The initial symptom is mostly nerve root pain, followed by paresthesia and motor dysfunction (5). The course of disease in this group was 4-36 months (13.5 months on average), but that of 6 cases with thoracic tumor was shorter for 4-9 months (5.4 months on average); it was easy to be misdiagnosed as cervical spondylosis due to the radiating pain in chest and back and weakness in walking in lower limb. Seventeen patients suffered from obvious limb radiating pain, night pain and supine pain. Paresthesia in different degrees was found in physical examination. Tumor tissues of 10 patients associated with the symptoms of weakness in walking were located in the cervical and thoracic segments, and physical examination showed the decreased muscle strength, tendon reflex active or hyperreflexia, and positive pathological signs; 2 patients with lumbosacral tumor suffered from numbness in saddle area and fecal and urinary dysfunction.
Early diagnosis is critical because the long-term spinal cord compression may lead to permanent loss of function. Detailed physical examination combined with MRI has a higher diagnostic value (6). MRI often showed intradural extramedullary circular, or dumbbell-shaped mass, T1WI showed the slightly lower signal, equal signal or equally-low mixed signal, and T2WI mostly showed high signal or mixed signal, and cystic change and necrosis might occur (7). In this group, 20 cases had intradural extramedullary oval mass with clear border, 6 cases had dumbbell-shaped tumors and 1 case had larger sacral tumor with irregular shape growing to the anterior pelvic cavity. Enhanced MRI scan mostly showed the uniform enhancement or ring and lace-like enhancement, and few showed the non-uniform enhancement; cystic necrosis area and spinal dura mater had no significant enhancement (8-10). In this group, 16 cases underwent MRI enhanced scan, among which 6 cases showed the significant enhancement and 10 cases showed the irregular edge enhancement. It is not difficult to be diagnosed as intraspinal tumor, but its nature is hard to be defined before operation, and intraspinal neurilemmoma should be distinguished from meningioma, neurofibroma, teratoma and metastatic tumor $(4,6)$. Pre-operative prediction of tumor nature is essential for intraoperative operation and post-operative recovery. In this group, the nature of 19 cases of tumors was correct before operation, and another 8 cases 
were difficult to diagnose, but confirmed pathologically after operation.

Intraspinal neurilemmoma should receive operation as soon as possible once diagnosed (11). Intraspinal neurilemmoma is a mostly benign tumor and surgical resection has a good effect with little recurrence after total resection (12). Surgical approach should be determined based on the tumor site and invasion range, and the semi-laminectomy on the affected side or total laminectomy retaining both sides of zygapophyseal joints or semi-laminectomy combined with facetectomy on the affected side should be performed. One case in this group received combined anterior and posterior approach due to the invasion of large tumor against anterior pelvic cavity. The intraoperative fluoroscopy is difficult for the upper-middle thoracic tumor, due to the blocking of scapula, so we think it is necessary to accurately position before surgery, and the corresponding parts should be often fixed with one to two clips for intraoperative positioning. The operation should be gentle to avoid excessive pulling of spinal cord nerve. High-speed abrasive drilling should be used for patients with cervical and thoracic tumors, and if the tumor is located in the spinal cord ventrally or ventral lateral region, the bone window should be made to fully expose tumor tissue (9). If the tumor is larger and the total resection is difficult, tumors can be resected in batches. For dumbbell-shaped tumors, it is necessary to fully remove the lamina and zygapophyseal joint on the affected side to fully expose the tumor and realize one-time resection $(13,14)$.

According to the current viewpoint, the tumor tissue should be totally resected as far as possible when tumorbearing nerves are treated. For thoracolumbar tumors, the pure disconnection of nerve roots generally does not cause new neurological deficits, but the cervical segment should be retained as far as possible (15). Schultheiss and Gullotta (16) found that patients did not suffer from serious functional loss after the tumor-bearing nerves were cut off. Celli (17) reported that a small number of patients suffered from permanent motor dysfunction after nerve roots in C5-C8 or L3-S1 were cut off. In this group with 5 cases of tumors, there was 1 case in cervical segment, 3 cases in thoracic segment and 1 case in lumbar segment; 1-2 nerve roots crossed with unclear boundary, and the tumor was totally resected; no obvious neurological dysfunction was found after operation.

In total laminectomy, the bony structures of posterior ligament complex and posterior column are resected, easily leading to iatrogenic spinal instability, so the internal fixation and bone graft fusion are needed to reduce the risk of post-operative spinal deformity $(18,19)$. According to the age and activity requirement, 11 patients receiving total laminectomy in this group adopted spinal dynamic pedicle screw fixation or replantation of lamina or bone graft fusion and internal fixation. Semi-laminectomy has less affect on spinal stability, so 10 patients receiving semilaminectomy in this group did not adopt the internal fixation, and no significant spinal instability was found during the follow-up for 48 months after operation. Moreover, 6 patients receiving semi-laminectomy combined with facetectomy on the affected side adopted the internal fixation.

Dural sac often needs to be opened in such surgery, so the cerebrospinal fluid leakage is more common after operation. Yang et al (20) reported their experience that the drainage and infection prevention time should be appropriately prolonged and drainage port should be locally sutured after drainage tube is removed. Ten patients suffered from cerebrospinal fluid leakage after operation, so the position was adjusted (Trendelenburg positionor dorsal elevated position), followed by wound dressing and pressure dressing (thick cotton cushion and elastic bandage could be used locally); the drainage time was prolonged appropriately, fluid infusion was enhanced and non-vacuum drainage was adopted. There were 3 cases of refractory cerebrospinal fluid leakage, and the wound was sutured in debridement and healed. We think that the prevention of cerebrospinal fluid leakage is very important, and the dural sac opened during operation should be closely and continuously sutured using 6-0 non-invasive stitches with the stitch controlled within $1 \mathrm{~mm}$. Multiple suture is not appropriate for patients with dural tear during operation, due to dural thinning and increased brittleness, because the tear will be larger; so the adjacent fascial flap can be used to cover the tear. When the incision is sutured, the muscle layer should be aligned closely without dead space, and the fascia, subcutaneous tissue and skin should be sutured layer by layer.

\section{Acknowledgements}

This study was supported by the Project of Invigorating Health Care through Science, Technology and Education (Jiangsu Provincial Medical Youth Talent) and Changzhou High-level Medical Talents Training Project (2016CZBJ029).

\section{References}

1. Weber C, Gulati S, Jakola AS, Habiba S, Nygaard ØP, Johannesen TB and Solheim O: Incidence rates and surgery of primary intraspinal tumors in the era of modern neuroimaging: A national population-based study. Spine 39: E967-E973, 2014.

2. Pan Z, Yang G, He H, Zhao G, Yuan T, Li Y, Shi W, Gao P, Dong L and $\mathrm{Li} \mathrm{Y}$ : Concurrent radiotherapy and intrathecal methotrexate for treating leptomeningeal metastasis from solid tumors with adverse prognostic factors: A prospective and single-arm study. Int J Cancer 139: 1864-1872, 2016.

3. Sowash M, Barzilai O, Kahn S, McLaughlin L, Boland P, Bilsky $\mathrm{MH}$ and Laufer I: Clinical outcomes following resection of giant spinal schwannomas: A case series of 32 patients. J Neurosurg Spine 26: 494-500, 2017.

4. Merhemic Z, Stosic-Opincal T and Thurnher MM: Neuroimaging of Spinal Tumors. Magn Reson Imaging Clin N Am 24: 563-579, 2016.

5. Safaee MM, Lyon R, Barbaro NM, Chou D, Mummaneni PV, Weinstein PR, Chin CT, Tihan T and Ames CP: Neurological outcomes and surgical complications in 221 spinal nerve sheath tumors. J Neurosurg Spine 26: 103-111, 2017.

6. dos Santos MP, Zhang J, Ghinda D, Glikstein R, Agid R, Rodesch G, Tampieri D and terBrugge KG: Imaging diagnosis and the role of endovascular embolization treatment for vascular intraspinal tumors. Neurosurg Focus 39: E16, 2015.

7. Masaryk TJ: Neoplastic disease of the spine. Radiol Clin North Am 29: 829-845, 1991.

8. Bendszus M, Urbach H, Wolf HK, Schramm J and Solymosi L: Magnetic resonance imaging of intraspinal melanotic schwannoma. Eur Radiol 8: 1197, 1998.

9. Lee RR: MR imaging of intradural tumors of the cervical spine. Magn Reson Imaging Clin N Am 8: 529-540, 2000.

10. Ahn DK, Park HS, Choi DJ, Kim KS, Kim TW and Park SY: The surgical treatment for spinal intradural extramedullary tumors. Clin Orthop Surg 1: 165-172, 2009.

11. Zong S, Wu Y, Tao Y, Chen X, Fang Y, Du L, Zhao J and Zeng G: Treatment results in different surgical approaches for intraspinal tumor in 51 patients. Int J Clin Exp Med 8: 16627-16633, 2015.

12. Viereck MJ, Ghobrial GM, Beygi S and Harrop JS: Improved patient quality of life following intradural extramedullary spinal tumor resection. J Neurosurg Spine 25: 640-645, 2016. 
13. Zhang Q, Ni M, Liu WM, Jia W, Jia GJ and Zhang JT: Intra- and extramedullary dumbbell-shaped schwannoma of the medulla oblongata: A case report and review of the literature. World Neurosurg 98: 873.e1-873.e7, 2017.

14. Iacopino DG, Giugno A, Gulì C, Basile L, Graziano F and Maugeri R: Surgical nuances on the treatment of giant dumbbell cervical spine schwannomas: Description of a challenging case and review of the literature. Spinal Cord Ser Cases 2: 15042 , 2016.

15. Das JM and Peethambaran A: Total excision of a giant ventral midline cervical spinal intradural schwannoma via posterior approach. Asian Spine J 10: 153-157, 2016.

16. Schultheiss R and Gullotta G: Resection of relevant nerve roots in surgery of spinal neurinomas without persisting neurological deficit. Acta Neurochir (Wien) 122: 91-96, 1993.
17. Celli P: Treatment of relevant nerve roots involved in nerve sheath tumors: Removal or preservation? Neurosurgery 51: 684-692, 2002

18. Yang P, He X, Li H, Zang Q and Wang G: Therapy for thoracic lumbar and sacral vertebrae tumors using total spondylectomy and spine reconstruction through posterior or combined anteriorposterior approaches. Oncol Lett 11: 1778-1782, 2016.

19. Hussein HA and Goda HA: Paravertebral neurogenic tumors with intraspinal extension: Preoperative evaluation and surgical approach. J Egypt Natl Canc Inst 21: 12-22, 2009.

20. Yang B, Wang Y, He X and $\mathrm{Li} \mathrm{H}$ : Treatment for thoracic ossification of posterior longitudinal ligament with posterior circumferential decompression: Complications and managements. J Orthop Surg 11: 153,2016 